\title{
Cariotipos Metafásicos de Drosophila inca y D. yangana, subgrupo inca, grupo repleta.
}

\author{
Ana Beatriz Mafla \\ Laboratorio de Genética, Escuela de Ciencias Biológicas, Pontificia \\ Universidad Católica del Ecuador. Apartado 17-01-2184. Quito-Ecuador. amafla@puce.edu.ec \\ Recibido 15 marzo, 2005. Aprobado 15 junio, 2005
}

\begin{abstract}
RESUMEN. Las especies del género Drosophila: $D$. inca y $D$. yangana pertenecientes al nuevo subgrupo inca del grupo de especies repleta son caracterizadas en términos de propiedades de los cromosomas metafásicos de ganglios cerebrales. En la descripción de los cariotipos se introduce la nomenclatura recomendada por Levan et al. (1964) con el objeto de precisar los rearreglos a nivel de las porciones heterocromáticas las cuales pasan desapercibidas en los análisis cariotípicos de cromosomas politénicos.
\end{abstract}

PALABRAS CLAVE. Cariotipos metafásicos, heterocromatina, subgrupo inca.

ABSTRACT. This work supply the information about the metaphase karyotypes of Drosophila inca and D. yangana; similarities and differences, introducing in the description the nomenclature recommended by Levan et al.(1964) it allow to detect arrangements at the level of the heterochromatin, which are unprovided in the polytene chromosomes.

KEYWORDS. Metaphase karyotypes, heterochromatin, inca subgroup.

\section{Introducción}

El subgrupo inca del grupo repleta está constituido por tres especies: Drosophila inca Dobzhansky \& Pavan, 1943 (1); D. huancavilcae Rafael \& Arcos, 1989 (2) y D. yangana Rafael \& Vela, 2003 (3), estas dos últimas descubiertas en el Ecuador. Se desconocen las particularidades biológicas de este nuevo subgrupo de especies; por lo que se ha abordado estudios que permitan su caracterización y eventualmente el establecimiento de las relaciones filogenéticas, tanto dentro del subgrupo inca, como con los miembros de los otros subgrupos.

Los ciclos de vida de $D$. inca y $D$. yanga$n a$, se han estudiado en condiciones de laboratorio, las dos especies presentan una duración promedio, desde la eclosión del huevo hasta la eclosión del adulto, de 22 días; aunque se observa que Drosophila inca tiene períodos de duración ligeramente menores en las etapas de huevo y primer estadio larvario y mayores en la fase de pupa (4).
En cuanto a la información citológica de Drosophila inca es muy limitada por eso se presenta en este trabajo los cariotipos metafásicos de $D$. inca junto a los de $D$. yangana: sus parecidos y diferencias, introduciendo en la descripción la nomenclatura recomendada por Levan et al. (5).

\section{Materiales y Métodos}

Las muestras analizadas corresponden a tres isolíneas de $D$. inca y tres de $D$. yangana fundadas a partir de hembras recolectadas en la naturaleza durante marzo del 2002 en la localidad andina de Yangana, provincia de Loja, Ecuador $\left(4^{\circ} 23^{\prime} \mathrm{S}, 79^{\circ} 11^{\prime} \mathrm{W}\right)$. Se disectaron larvas de tercer estadio para extraer los ganglios cerebrales, en un portaobjeto excavado se colectaron de 10 a 12 ganglios cerebrales en solución de Ringer-Drosophila, se transfirieron a otro porta excavado conteniendo una solución de Ringer: colchicina $0,01 \mathrm{M}$ en proporción $2: 1$ por 30 minutos; se extrae esta solución y se la 
remplaza por $\mathrm{KCl} 0,075 \mathrm{M}$ durante 20 minutos. Después del tratamiento hipotónico se fija el tejido en metanol: ácido acético (3:1) por mínimo 30 minutos al cabo de los cuales se extrae el fijador y se dispersa el tejido en ácido acético $60 \%$, se añade dos gotas de fijador y se gotea en portaobjetos los cuales fueron mantenidos en etanol y en refrigeración; las placas con la suspensión se dejan secar en una plancheta a $65^{\circ} \mathrm{C}$ (el shock térmico permite una mejor dispersión de los cromosomas). Las placas fueron teñidas con Giemsa diluído en agua desionizada en proporción 1:20.

Los mejores núcleos fueron fotografiados usando el lente de inmersión Neofluar 100, oculares $8 \mathrm{X}$ y aumento 1,25 de la cámara fotográfica obteniéndose una amplificación total de 1000X.

Los cariotipos se montaron recortando el perfil de los cromosomas en fotografias convencionales, se midieron los cromosomas y se organizaron en orden descendente. En la identificación de la forma cromosómica se sigue las recomendaciones de Levan et al. (1964); nominando a los cromosomas st, sm o $\mathrm{T}$ de acuerdo a la posición del centrómero en la región subterminal, submedial o en el punto Terminal, respectivamente. Los idiogramas se construyeron normalizando los valores de 20 cariotipos

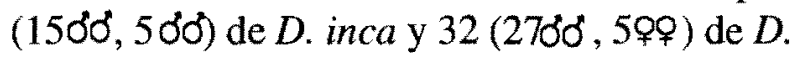
yangana. La normalización se realizó calculando la longitud relativa de cada cromosoma y luego promediando los valores de cada par homólogo, excepto para el par XY; igual procedimiento se aplicó en la normalización del índice centrómerico.

\section{Resultados y Discusión}

El número diploide en las dos especies es igual; $2 n=12$. Tomando como referencia el cariotipo de $D$. inca que fuera descrito por Dobzhansky \& Pavan, 1943 como compuesto de $5 \mathrm{R} 1 \mathrm{~J}^{1}$ podemos decir que los resultados obtenidos en este trabajo muestran coincidencia parcial, puesto que en las placas mitóticas obtenidas por suspensión celular se ha podido definir con mayor precisión la posición del centrómero, de modo que de las 5 varillas reportadas precisamos que en $D$. inca del Ecuador los cromosomas más grandes son st y los pequeños son $\mathrm{sm}$.

Los cariotipos fueron construidos ordenando los cromosomas por tamaño decreciente y reuniendo en el grupo A los 3 cromosomas más grandes: dos pares de autosomas y el par sexual heteromórfico, en este conjunto resalta el cromosoma Y por su heteropicnosis en tanto que el X se confunde con los demás. En el grupo $\mathrm{B}$ se reúne a los tres autosomas pequeños; en este conjunto es claramente identificable el par autosómico 6 por su menor tamaño y heteropicnosis.

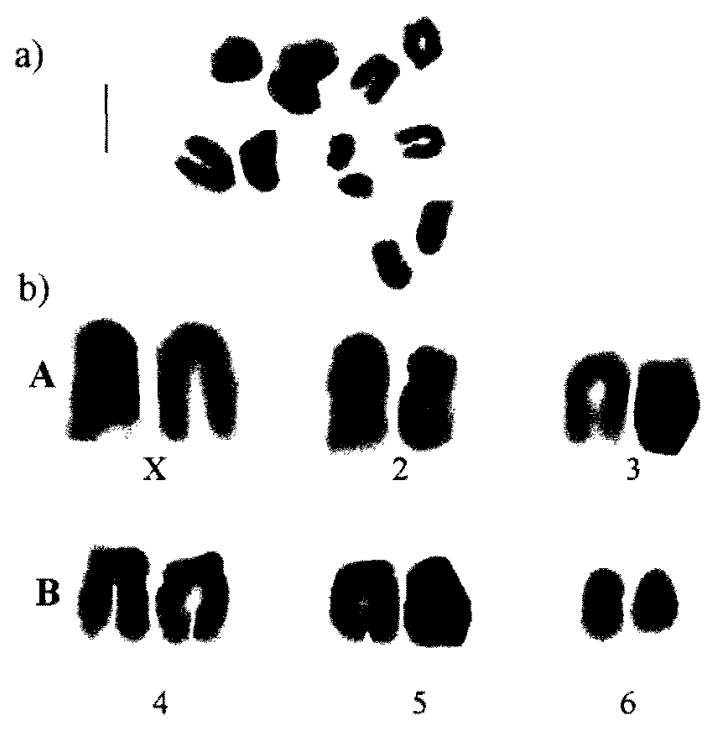

Fig. 1 a-b. a) Núcleo mitótico de ganglio cerebral de Drosophila inca $Q$, b) Cariotipo mostrando los grupos cromosómicos A y B. La barra corresponde a $4 \mu$.

En $D$. inca se evidencian tres pares de cromosomas st y $3 \mathrm{sm}$ (Fig. 1a, b). El grupo A con cromosomas cuyas longitudes relativas (LR) están entre 11,35 a 9,07; el más grande corresponde al cromosoma sexual $\mathrm{X}$, seguido del autosoma 2 con LR 9,92; y el autosoma 3 con LR de 9,07; estos tres cromosomas tienen el centrómero en posición st. El grupo B reúne

\footnotetext{
${ }^{1}$ Del inglés R: Rods = varillas; $\mathrm{J}$ = forma de esta letra; en la nomenclatura clásica de cromosomas de Drosophila .
} 
Tabla 1. Longitud relativa y morfología de los cromosomas de Drosophila inca y $D$.

yàngana

\begin{tabular}{|c|c|c|c|c|c|c|c|c|}
\hline & \multicolumn{4}{|c|}{ Grupo A } & \multicolumn{3}{|c|}{ Grupo B } & \multirow[b]{2}{*}{$\bar{B}$} \\
\hline & $\bar{X}$ & 2 & $\mathbf{Y}$ & 3 & 4 & 5 & 6 & \\
\hline \multirow[t]{2}{*}{ D. inca } & 11,35 & 9,92 & 9,21 & 9,07 & 8,32 & 7,08 & 5,08 & - \\
\hline & st & st & sm & st & sm & sm & sm & \\
\hline \multirow[t]{2}{*}{ D. yangana } & $10,19_{2 a}$ & 10,94 & 10,06 & 9,58 & 8,90 & 7,73 & 2,58 & 2,14 \\
\hline & sm & sm & sm & sm & sm & sm & $T$ & $\mathrm{~T}$ \\
\hline
\end{tabular}

a los 3 autosomas más pequeños con centrómero sm y cuyas LR están entre 8,32 y 5,08 ; el autosoma 6 aparece fuertemente heteropicnótico y su longitud corresponde a poco más de la mitad de la longitud del cromosoma más grande $(X)$.

En orden decreciente el heterocromosoma Y ocupa la tercera posición con LR 9,21 y centrómero sm. (Tabla 1).

En $D$. yangana hay 5 pares sm y uno $\mathrm{T}$ (Fig.2 a, b). El cromosoma más grande corresponde al autosoma 2 con LR de 10,94 seguido por el cromosoma X con una LR de 10,19; y el autosoma 3 con LR 9,58, todos los del grupo A tienen el centrómero sm. El grupo $\mathrm{B}$ comprende a los autosomas 4 y 5 de centrómero también sm y de LR 8,90 y 7,73 y al 6 T y LR 2,58 ; este último también es heteropicnótico y su tamaño corresponde a un cuarto de la del cromosoma más grande (autosoma 2).

En los cariotipos de D. yangana el heterocromosoma Y de LR 10,06 también ocupa la tercera posición (Tabla 1); es heteropicnótico con centrómero sm y presenta una constricción secundaria en el tercio distal del brazo largo (Fig. 3b). Llama la atención la coincidencia de este marcador con lo reportado para $D$. uniseta y D. starmeri miembros del subgrupo mulleri $(6,7)$; otro subgrupo del grupo repleta.

Además en $D$. yangana se han registrado núcleos con uno o dos cromosomas supernumerarios de LR 2,14, posición centromérica $T$ y heteropicnóticos. (Tabla 1).
Comparado con el cariotipo de $D$. inca el de $D$. yangana presenta las siguientes diferencias (Fig. 3) : $1^{\circ}$ el cromosoma $X$ ocupa la segunda posición en orden decreciente, $2^{\circ}$ los miembros del grupo A tienen centrómeros $\mathrm{sm}$; $3^{\circ}$ en el grupo B el autosoma 6 es $\mathrm{T}$ y corresponde a la mitad del tamaño del de $D$. inca, en las placas metafásicas aparece como un punto; $4^{\circ}$ resalta la presencia de cromosomas supernumerarios que son considerados excepcionales en el género Drosophila; el cromosoma B sumado al cromosoma 6 da una longitud correspondiente al 6 de D. inca (Tabla 2).

a)

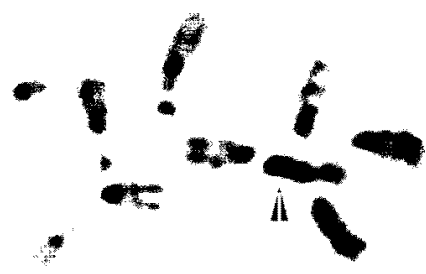

b)

A

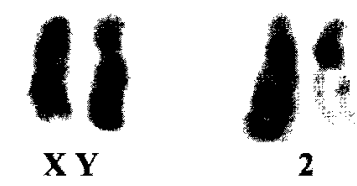

B

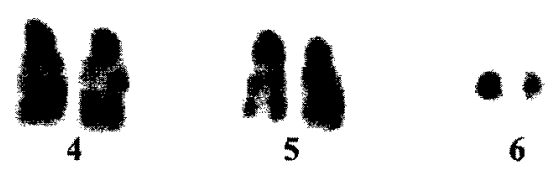

Fig. 2 a-b. a) Núcleo mitótico de Drosophila yangana d, b) Cariotipo mostrando los grupos cromosómi$\cos$ A y B. La barra corresponde a $4 \mu$. La flecha señala la constricción secundaria del cromosoma $Y$. 
a)

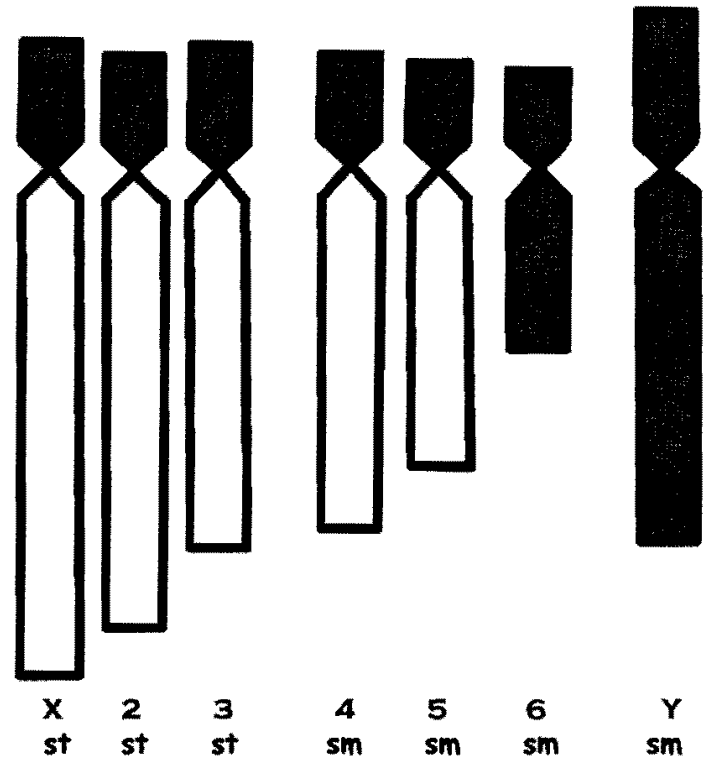

b)
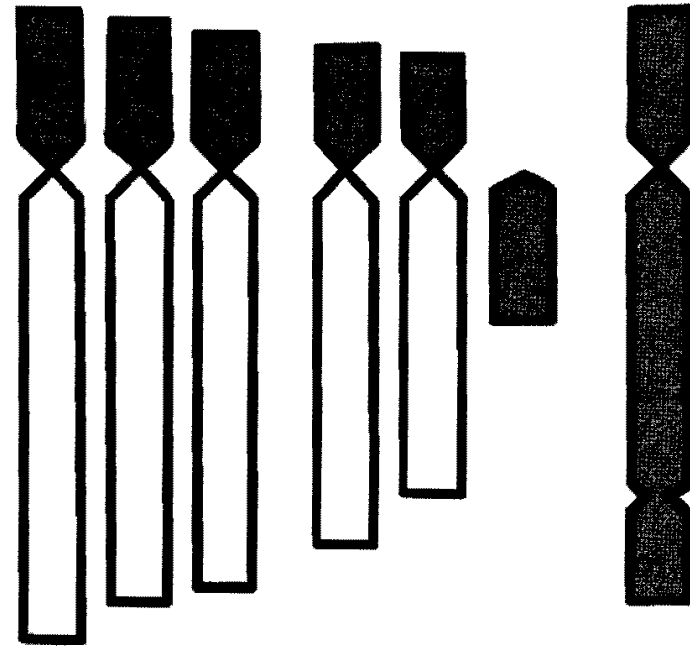

$\begin{array}{ccccccc}2 & X & 3 & 4 & 5 & 6 & Y \\ 5 m & 5 m & s m & s m & s m & T & s m\end{array}$

Fig. 3 a-b. Idiogramas de a) Drosophila inca, grupo A cromosomas st. Autosoma $6 \mathrm{sm}$. b) Drosophila yangana, grupo A cromosomas sm. Autosoma $6 \mathrm{~T}$.

Tabla 2. Comparación del porcentaje de heterocromatina en los cromosomas de Dinca y

$$
\text { D. yangana }
$$

\begin{tabular}{|c|c|c|}
\hline & Drosophila inca & D. yangana \\
\hline Cromosoma & & \\
\hline $\mathrm{X}$ & 4,84 & 5,32 \\
\hline 2 & 4,19 & 5,63 \\
\hline 3 & 4,33 & 4,75 \\
\hline 4 & 4,29 & 4,62 \\
\hline 5 & 3.79 & 4,23 \\
\hline 6 & 10,00 & 5,17 \\
\hline B & & 4,30 \\
\hline Y & 15,35 & 16,77 \\
\hline Total & $46,79 \%$ & $50,79 \%$ \\
\hline
\end{tabular}


Las dos especies son semejantes en la morfología de los autosomas 4 y 5 , del sexual $Y$ que ocupa la tercera posición, así como la heteropicnosis de los cromosomas Y y 6.

Como lo señala White (8) en Drosophila los cromosomas mitóticos permiten detectar rearreglos a nivel de la heterocromatina, los cuales pasan desapercibidos en los cariotipos de cromosomas politénicos; en este sentido, la presente descripción de los cariotipos de las dos especies de Drosophila, usando la nomenclatura recomendada por Levan et al. (1964) nos permite discernir que los brazos cortos de los cromosomas X, 2, 3, 4 y 5 de $D$. inca, contienen menor proporción de heterocromatina que los correspondientes de D. yangana. Sumando las longitudes de las porciones heterocromáticas (Tabla 2) podemos estimar el contenido total de cada genoma, en $D$. inca tenemos un valor de $46,79 \%$ en tanto que en $D$. yangana llega a $50,79 \%$.

\section{REFERENCIAS}

1.- DOBZHANSKY, TH. \& PAVAN, C. Studies on Brazilian Species of Drosophila. Separata do Bol. Fac. Fil. Ciên. E Letr. Univ. S. Páulo, $N^{\circ}$ 36-Biologia Geral 1943; $N^{\circ} 4$ : 44-46.

2.- RAFAEL, V. \& ARCOS, G.. Subgrupo inca, un nuevo subgrupo del grupo repleta, con descripción de Drosophila huancavilcae nov. sp. (Diptera, Drosophilidae). Evolución Biológica, Bogotá, 1989; 3 (3): $233-243$.

3.- RAFAEL, V. \& VELA, D. Drosophila yangana sp. nov. un nuevo miembro del grupo repleta, subgrupo inca (Diptera: Drosophilidae). Revista de la Pontificia Universidad Católica del Ecuador, Quito, 2003; 71:129-139.

4,- MAFLA, A. B. Life cycles and fitness components of Drosophila inca and D. yangana (Diptera, Drosophilidae) Iheringia, Sér. Zool., Porto Alegre, 2005; 95 (1); 89-91.

5.- LEVAN, A.; FREDGA, K. \& SANDBERG, A.A. Nomenclature for centromeric position on chromosomes. Hereditas, 1964; 52 (2).

6.- WASSERMAN, M. \& KOEPFER, H. R. Cytogenetics of the South American Drosophila mulleri Complex: The martensis cluster, more interspecific sharing of inversions. Geneties 1979; 93: 935-946.

7.- WASSERMAN, M. Evolution of the repleta Group. In: The Genetics and Biology of Drosophila. AsHBuRNER, M., Carson, H. L. \& Thompson, J. N. JR., eds. Academic Press, London, 1982; 3b: 61-139.

8.- WHITE, M.J.D. Modes of Speciation. W.H.Freeman and Company, San Francisco, 1978, pg. 66. 